\title{
Optical bend sensor for vector curvature measurement based on Bragg grating in eccentric core polymer optical fibre
}

\author{
X. Chen ${ }^{1}$, C. Zhang ${ }^{1}$, D. J. Webb ${ }^{1}$, R. Suo ${ }^{1}$, G. D. Peng ${ }^{2}$, K. Kalli ${ }^{3}$ \\ ${ }^{1}$ Photonics Research Group, Aston University, Birmingham B4 7ET, UK \\ ${ }^{2}$ School of Electrical Engineering, University of New South Wales, Australia \\ ${ }^{3}$ Nanophotonics Research Laboratory, Higher Technical Institute, Cyprus
}

\begin{abstract}
We present an optical bend sensor based on a Bragg grating written in an eccentric core polymer optical fibre. The grating wavelength shifts are studied as a function of bend curvature and fibre orientation and the device exhibits strong fibre orientation dependence, wide bend curvature range of $\pm 22.7 \mathrm{~m}^{-1}$ and high bend sensitivity of $63 \mathrm{pm} / \mathrm{m}^{-1}$, which is 80 times higher than the reported sensor based on an offset-FBG in standard single mode silica fibre.
\end{abstract}

Keywords: Polymer optical fibre, Bragg grating, bend sensor

\section{INTRODUCTION}

Since Hill et al. first reported the photosensitivity of silica optical fibre [1], fibre Bragg gratings (FBGs) and long-period gratings (LPGs) have been widely developed and utilized as devices for optical communications and optical sensing [2, 3]. An important application of fibre gratings is in the measurement of curvature. To date, a number of bend sensors have been realized using LPGs, where the bending of the fibre induces the wavelength shifts or bend splitting of the LPGs' spectral attenuation bands [4-6]. However, the full width at half maximum (FWHM) of an LPG resonance is typically tens of nanometers, which limits the measurement accuracy. In addition, the conventional LPG length is tens of millimeters which compromises the spatial resolution achievable. In the past decade, several bend sensors based on FBGs in multi-core fibre or the axially-offset FBG in single mode silica fibre have been reported [7, 8]. Recently, gratings written in polymeric optical fibres (POFs) have begun to attract interest since the first report of Xiong et al. [9]. In comparison with silica fibre, single mode POF is more difficult to work with in many regards but has some attractive features, such as a much smaller Young's modulus and a much greater breaking strain. POF-based FBGs have demonstrated a large dynamic range for temperature and strain sensing [10-13].

This paper reports on a study of the bend sensitivity of a Bragg grating written in eccentric core polymer optical fibre (ecPOF) with large core offset. This sensor has been used to measure the vector curvature, showing high bend sensitivity, wide bend curvature range and strong directional dependence.

\section{FBG FABRICATION AND SPECTRUM MEASUREMENT}

As can be seen from the microscope image of the cross-section in Fig. 1, the polymer fibre used in our experiments has a circular geometric cladding with a diameter of $230 \mu \mathrm{m}$ and two eccentric cores, which are offset from the cladding centre, i.e., the fibre axis. In the work reported here, only one eccentric core, which is closest to the fibre axis and marked as "ec1" in Fig.1, was chosen for grating fabrication and then for curvature sensing. The diameter of this core is $8.1 \mu \mathrm{m}$ and the core offset from the fibre axis is $24 \mu \mathrm{m}$. We attached small masking tapes to the fibre to indicate the fibre at $0^{\circ}$ orientation, where the fibre axis and 'ec1' core lie along the same line.

The FBG was written using a CW helium-cadmium laser with an output wavelength of $325 \mathrm{~nm}$, a beam diameter of $1.8 \mathrm{~mm}$ and a power of $30 \mathrm{~mW}$. The ecPOF was supported along its entire length by a v-groove during fabrication to keep the polymer fibre straight. The writing beam was focused vertically downwards using cylindrical lens of focal length $100 \mathrm{~mm}$ to expose the ec1-core. A $4 \mathrm{~mm}$ long FBG was inscribed through a phase mask with period of $1.057 \mu \mathrm{m}$ and scanned four times with a speed of $0.05 \mathrm{~mm} / \mathrm{s}$.

*x.f.chen@aston.ac.uk; Phone: +44 (0) 121 2043518; Fax: +44 (0) 1212043682

20th International Conference on Optical Fibre Sensors, edited by Julian Jones, Brian Culshaw,

Wolfgang Ecke, José Miguel López-Higuera, Reinhardt Willsch, Proc. of SPIE Vol. 7503, 750327

(c) 2009 SPIE $\cdot$ CCC code: $0277-786 X / 09 / \$ 18 \cdot$ doi: $10.1117 / 12.834958$

Proc. of SPIE Vol. $7503750327-1$ 


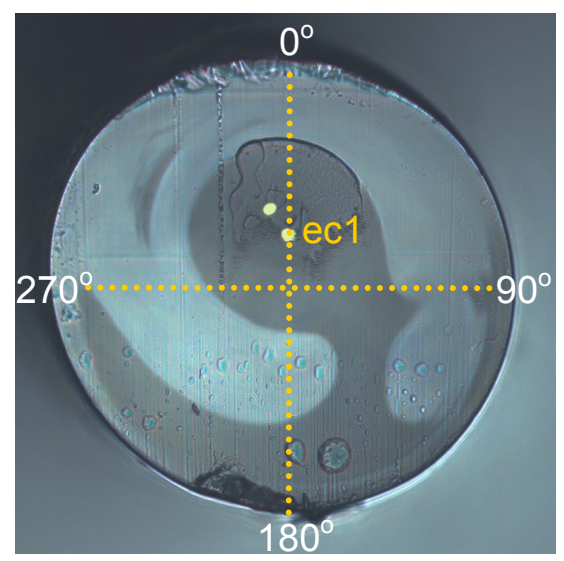

Fig. 1 Microscope image of cross section of the cleaved ecPOF end marked with the orientations.

(Note that only the "ec1" core was used for FBG fabrication for bend sensing.)

The grating reflection spectra were monitored using a single mode silica fibre 3-dB coupler, a broadband light source (BBS) and an optical spectrum analyser (OSA) in conjunction with a three-dimensional stage for butt-coupling. Both ends of ecPOF were cut using an $85^{\circ} \mathrm{C}$ heated sharp razor blade to create a flat end face. The light was butt-coupled from one arm of the coupler to the ecPOF cleaved-end by adjusting the three-dimensional stage. In order to ensure the light was launched into the core containing the FBG by the butt-coupling, an IR camera was used to view the near field image of the ecPOF output end face to check the exact coupling position. Fig. 2 shows two near field images of the ecPOF output with the light coupled in two eccentric cores simultaneously (a), and then only coupled in the chosen core containing the FBG (b).

In order to eliminate back reflection from the fibre ends, refractive index matching gel was applied. In addition, a small amount of refractive index gel was added between the ends of the silica fibre and ecPOF to minimise any Fresnel reflections and therefore improve the light coupling. When the light was launched in the core shown as Fig. $2 \mathrm{~b}$, the grating reflection was coupled back into the addressing fibre and was monitored by the OSA with the resolution bandwidth of $0.06 \mathrm{~nm}$. The reflection spectrum is plotted in Fig. 2c displaying a Bragg wavelength of $1562.5 \mathrm{~nm}$, a FWHM of $0.49 \mathrm{~nm}$, and a reflective power above the noise level of $10.5 \mathrm{~dB}$.

(a)

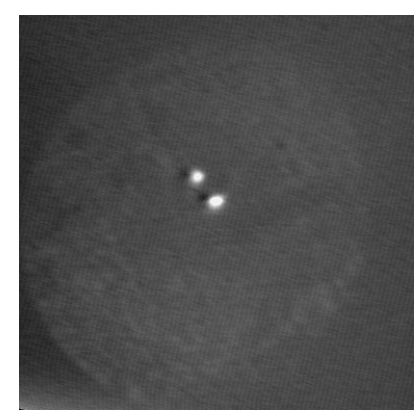

(b)

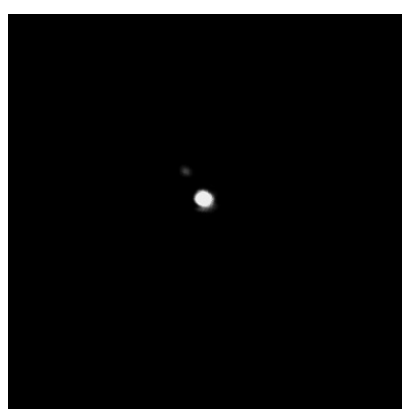

(c)

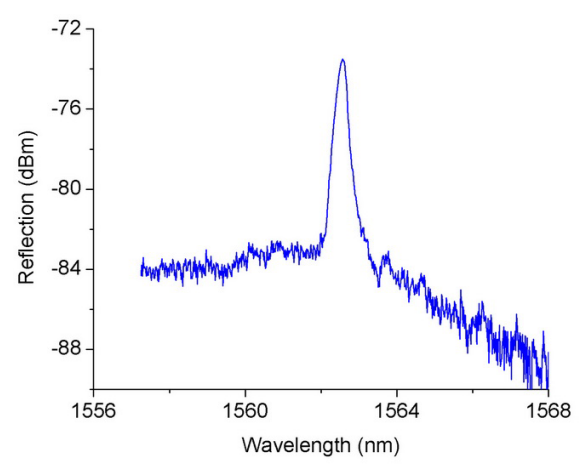

Fig. 2 Near-field images at the output end-face of ecPOF under different butt-coupling situations: (a) to both of the eccentric cores, (b) to the selected eccentric core containing the grating. The spectrum reflected by the FBG is shown in (c).

\section{CONFIGURATION OF BEND SENSING SYSTEM}

The experimental arrangement for vector curvature measurement is illustrated in Fig.3. The polymer fibre was hung between two stages with the FBG at the centre of the $9 \mathrm{~cm}$ long bend section, resulting in a bend that lay in the vertical plane, i.e., y-z plane. Control of the bend was achieved by the translation of the right-hand end of the polymer fibre, 
which was driven by a micrometer screw along '- $\mathrm{z}$ ' direction. The bending radius $\mathrm{R}$ of the polymer fibre is calculated based on the equation as $\mathrm{R}=\left(\mathrm{h}^{2}+\mathrm{l}^{2}\right) / 2 \mathrm{~h}$, thus, the curvature $\mathrm{C}$ is given by [5]

$$
C=\frac{1}{R}=\frac{2 h}{h^{2}+l^{2}}
$$

where $h$ is the displacement in y-z plane from the idle position, $l$ is the half length of fibre bend section. The grating central wavelength was monitored as a function of curvature.

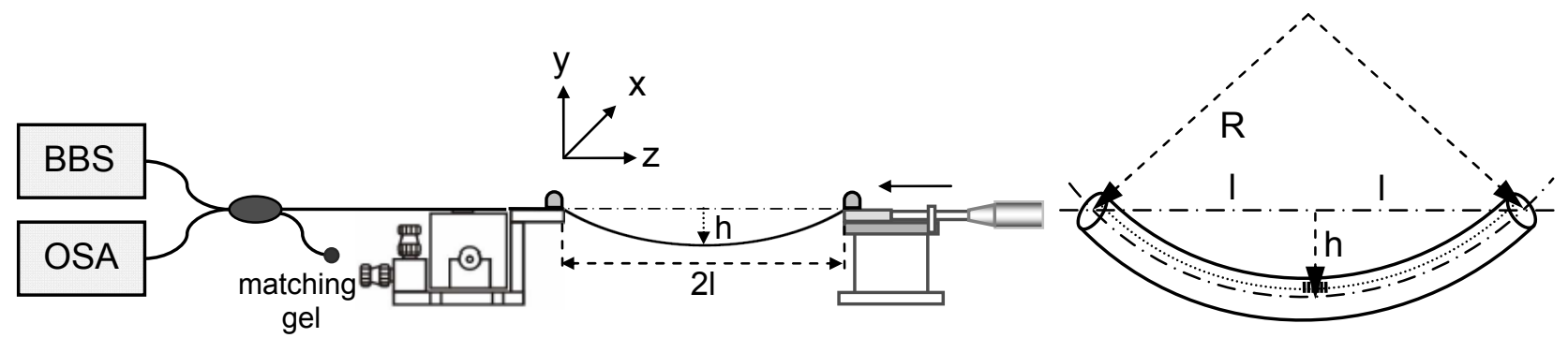

Fig. 3 Schematic of the experimental setup for implementation of FBG based bending sensor.

\section{EXPERIMENTAL RESULTS AND DISCUSSION}

Experiments were conducted to determine the reflection spectral responses of the ecPOF grating to variations of curvature for different fibre orientations. Small tape tags, attached to the ecPOF with an initial orientation at $0^{\circ}$, were used to indicate rotation of fibre with respect to the vertical plane. The bend experiment started with $0^{\circ}$-orientation, then the ecPOF was rotated by $90^{\circ}$, and the bend test repeated; this was continued until response had been mapped over the four orientations. For each rotational orientation, the reflection spectrum was monitored for a curvature rang from 0 to $22.7 \mathrm{~m}^{-1}$. Strong directional bend sensitivity characteristics were observed in the ecPOF when bending was applied at different fibre orientations. With the ecPOF fixed at $0^{\circ}$-orientation, the reflection spectra shifted toward shorter wavelengths (Fig. 4a). In contrast, the spectra moved to longer wavelengths for the $180^{\circ}$-orientation case (Fig. 4b).

(a)

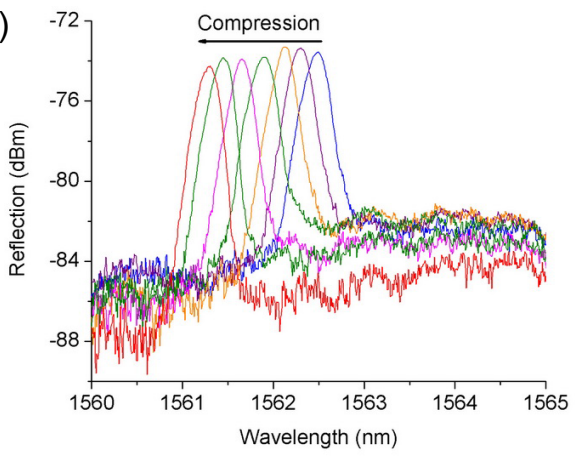

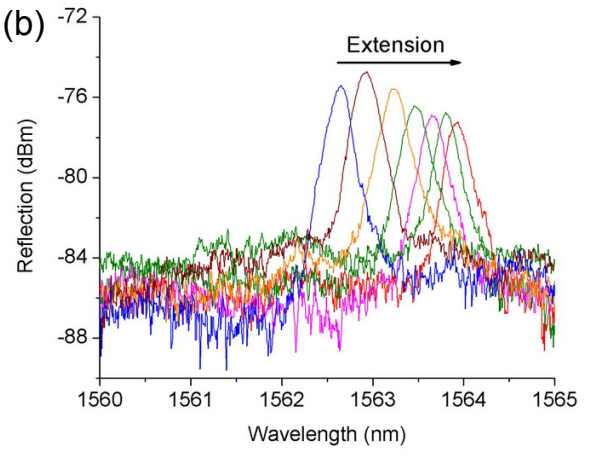

Fig. 4 Spectral response of the FBG in ecPOF experiencng bending at different fibre orientation: (a) $0^{\circ}$, (b) $180^{\circ}$.

The Bragg wavelength shifts of the grating in ecPOF against curvature for four fibre orientations are quantified in Fig. 5a. It is clear that the bend responses are directionally sensitive and all responses exhibit near linear features. It has been demonstrated that the maximum bend response occurred at $0^{\circ}$ and $180^{\circ}$ orientation where the eccentric grating and fibre axis lie in the bend plane. In addition, $0^{\circ}$ and $180^{\circ}$ orientation showed the opposite bend response. It may be explained with the schematic drawing in Fig. 5b. Because the eccentric grating is axially-offset from the fibre neutral axis, when the grating was on the inside of the bend $\left(0^{\circ}\right.$ orientation case), it experienced a compressive strain whereas an outside bend $\left(180^{\circ}\right.$ orientation case) led to an extension. For $90^{\circ}$ and $270^{\circ}$ orientation, the grating and fibre axis lay in the same neutral bending plane, therefore the minimum response was obtained, showing for low curvatures a bend insensitive property. 


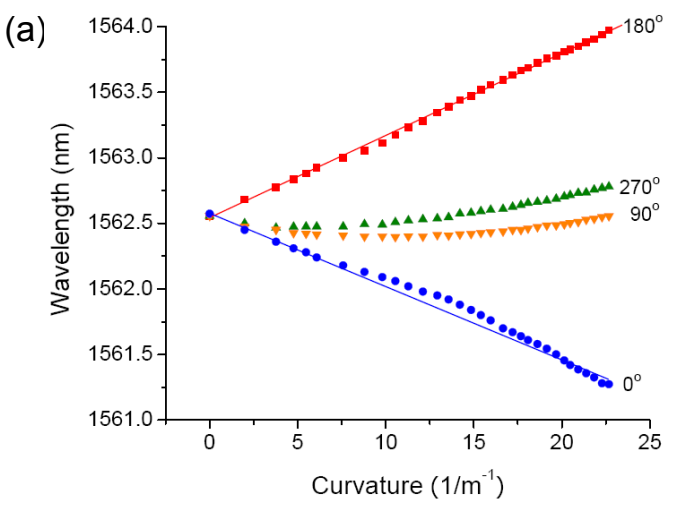

(b)

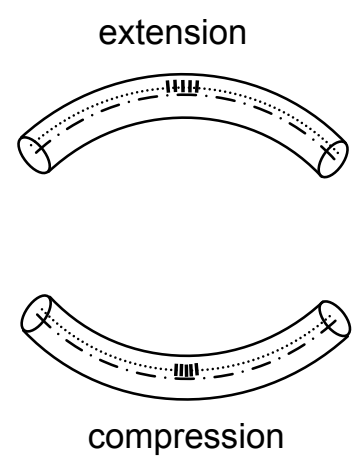

Fig. 5 (a) Wavelength shifts of the eccentric grating in polymer fibre against curvature at different orientations;

(b) Schematic showing bending leading to compression and extension.

The core is offset from the fibre axis, inducing the required asymmetry in the device and therefore inducing directional bending sensitivity: with a $22.7 \mathrm{~m}^{-1}$ curvature compression bend $\left(0^{\circ}\right.$ orientation), a blue wavelength shift of $-1.26 \mathrm{~nm}$ was obtained; with the same magnitude of extension bend $\left(180^{\circ}\right.$ orientation), there was a red wavelength shift of $+1.42 \mathrm{~nm}$; with the fibre orientation rotated to $90^{\circ}$ or $270^{\circ}$, the wavelength shift was within $\pm 0.18 \mathrm{~nm}$. The departure from linearity observed in Fig. 5 and the sensitivity to bending observed at the $90^{\circ}$ and $270^{\circ}$ positions may be caused by non-perfect circular geometry of the POF cladding, misalignment of the grating axis with the $0^{\circ}$ bend plane or a slight twist of the POF during bend measurement. The best-fit straight lines to the experimental data in Fig.5 give a negative gradient for compression mode and positive for extension mode: $-56 \mathrm{pm} / \mathrm{m}^{-1}$ and $+63 \mathrm{pm} / \mathrm{m}^{-1}$, respectively. In this work, the grating axial-offset is $24 \mu \mathrm{m}$ which is 8 times that used in an experiment with silica fibre [8], but the maximum bend sensitivity here of $63 \mathrm{pm} / \mathrm{m}^{-1}$ is 80 times higher than the silica device. In our work, the polymer FBG can respond to a curvature larger than $22.7 \mathrm{~m}^{-1}$ with the width of the FBG resonance still narrow enough for accurate measurement.

\section{CONCLUSIONS}

In summary, we have demonstrated a vector curvature sensor based on a Bragg grating inscribed in an eccentric core polymer fibre. This polymer grating sensor is capable of measuring the magnitude and orientation of the applied bend. The maximum bend sensitivity achieved, $63 \mathrm{pm} / \mathrm{m}^{-1}$, is 80 times higher than that of silica FBG bend sensor, showing significant advantages over the silica gratings. One issue with devices of this kind is how to produce a simple means of coupling light from a conventional fibre into the eccentric core; the POF production process in principle allows the development of fibre in which the core alternates from being concentric to being eccentric along the fibre length. This offers the possibility of coupling to the POF where the core is concentric but still having an eccentric sensing region.

\section{ACKNOWLEDGEMENT}

EU FP7 Project PHOSFOS (PHOtonic Skins For Optical Sensing) and UK Engineering and Physical Sciences Research Council.

\section{REFERENCES}

K. O. Hill, Y. Fujii, d. C. Johnson, and B. S. Kawasaki, Appl. Phys. Lett. 32, 647-649, 1978.

A. Othonos and K. Kalli, Fibre Bragg Gratings, (Artech House, 1999).

T. Erdogan, J. Lightwave Technol. 15, 1277-1294, 1997.

H. J. Patrick, Electron. Lett., 36 (21), 1763-1764, 2000.

Y. Liu, L. Zhang, J. Williams, and I. Bennion, IEEE Photon. Technol. Lett., 12 (5), 531-533, 2000.

D. Zhao, K. Zhou, X. Chen, L. Zhang, I. Bennion, W. Macpherson, J. Barton, and J. Jones, Meas. Sci. Technol. 15, 1647-1650, 2004.

G. M. H. Flockhart, W. N. MacPherson, J. S. Barton, J. D. C. Jones, L. Zhang, I. Bennion, Opt. Lett., 28 (6), $387-389,2003$.

A. Martinez, Y. Lai, M. Dubov, I. Y. Khrushchev, and I. Bennion, Electron. Lett., 41(8), 472-474, 2005.

Z. Xiong, G. D. Peng, B. Wu, and P. L. Chu, IEEE Photon. Technol. Lett., 11(3), 352-354, 1999.

0 H. B. Liu, H. Y. Liu, G. D. Peng, and P. L. Chu, Opt. Commun., 219, 139, 2003.

1 K. E. Carroll, C. Zhang, D. J. Webb, K. Kalli, A. Argyros, and M. C. J. Large, Opt. Express. 15 (14), 8844-8850, 2007.

12 H. Dobb, D. J. Webb, K. Kalli, A. Argyros, M. C. J. Large, and M. A. Eijkelenborg, Opt. Lett. 30(24), 3296-3298, 2005.

13 Y. Luo, J. Zhou, Q. Yan, W. Su, Z. Li, and Q. Zhang, Appl. Phys. Lett. 91, 071110, 2007. 